\title{
Parâmetros da pressão plantar, tipo e sensibilidade do pé em recrutas: um estudo prospectivo
}

\author{
Plantar pressure parameters, type and sensitivity of the foot in recruits: a prospective study \\ Parámetros de la presión plantar, tipo y sensibilidad de pies de reclutas: un estudio prospectivo \\ Andressa Hardt de Jesus ${ }^{1}$, Fernando Copetti ${ }^{2}$, Michele Forgiarini Saccol ${ }^{3}$
}

\begin{abstract}
RESUMO I Este estudo teve como objetivo avaliar as características da pressão plantar, tipo e sensibilidade do pé em recrutas durante o período do serviço militar obrigatório. Sessenta indivíduos que prestaram o serviço militar obrigatório foram avaliados para pressão plantar (baropodômetro EPS LoranEngineering, Bolonha, Itália) e sensibilidade plantar ao toque superficial (estesiômetro de Semmes-Weinsten) em três momentos distintos: março (linha de base), junho (após 16 semanas de treinamento) e setembro (após 36 semanas de treinamento militar). Foram avaliadas a descarga de peso ântero-posterior e laterolateral, pressão média, pressão máxima, área de contato do pé, tipologia de pé e sensibilidade plantar. Os dados foram analisados pelo teste do Qui-quadrado, anova de medidas repetidas e pelo teste de Friedman com pos hoc deBonferroni $(p<0,05)$. Houve aumento na área de contato $(p=0,001)$ e na pressão média de ambos os pés $(p<0,001)$. Quanto a tipologia, aproximadamente $60 \%$ dos indivíduos possuíam o pé direito do tipo normal, enquanto o pé esquerdo era do tipo cavo (50,3\%); ou seja, mesmos sujeitos apresentaram diferentes tipologias do pé. Em relação a sensibilidade plantar, houve diferença na região do médio pé ao longo do treinamento $(p=0,001$ no pé dominante e $p=0,009$ no pé não dominante). Esses resultados demonstram que houve aumento da pressão média e área total de contato do pé, bem como alterações na sensibilidade plantar da região do médio pé ao longo do serviço militar obrigatório.
\end{abstract}

Descritores | Pé; Pressão; Mecanorreceptores.

ABSTRACT I This study aimed to evaluate the characteristics of plantar pressure, type of foot and plantar sensitivity of recruits during the period of compulsory military service.
Sixty individuals who performed compulsory military service were assessed for plantar pressure (EPS LoranEngineering baropodometer, Bologna, Italy) and plantar sensitivity to superficial touch (esthesiometer - Semmes-Weinsten Monofilaments) at three different times: March (baseline), June (after 16 weeks of training) and September (after 36 weeks of military training). Antero-posterior and laterolateral weight bearing, mean pressure, maximum pressure, foot contact area, foot typology were evaluated. and plantar sensitivity. The data were analyzed using the chi-squared test, Anova repeated measures and Friedman test with Bonferroni pos hoc $(p<0,05)$. There was an increase in contact area $(p=0,001)$ and mean pressure of both feet $(p<0,001)$. As for the typology, an average of $60 \%$ of the individuals have the dominant foot of the normal type, while the non-dominant foot are cavus (50.3\%), that is, the same subjects presented different typologies of the foot. Regarding plantar sensitivity, there was a difference in the midfoot region over training time $(p=0.001$ in the dominant foot and $p=0.009$ in the non-dominant foot). These results demonstrated that there was an increase in average pressure and total foot contact area, and also plantar sensitivity alterations throughout the mandatory military period. Keywords | Foot; Pressure; Mechanoreceptor.

RESUMEN I Este estudio tuvo como objetivo evaluar las características de la presión plantar, el tipo de pie y la sensibilidad en los reclutas durante el período de servicio militar obligatorio. Sesenta individuos que realizaron el servicio militar obligatorio fueron evaluados para presión plantar (baropodómetro EPS LoranEngineering, Bolonia, Italia) y sensibilidad plantar al tacto superficial (estesiómetro 
Semmes-Weinsten) en tres momentos diferentes: marzo (línea base), junio (después de 16 semanas de entrenamiento) y septiembre (después de 36 semanas de entrenamiento militar). Se evaluaron descarga de peso anteroposterior y laterolateral, presión media, presión máxima, área de contacto del pie, tipología del pie y sensibilidad plantar. Los datos se analizaron mediante el test de chi-cuadrado, el ANOVA de medidas repetidas y el test de Friedman con Bonferroni pos hoc $(p<0,05)$. Hubo un aumento en el área de contacto $(p=0,001)$ y en la presión media de ambos pies ( $p$ $<0,001)$. En cuanto a la tipología, aproximadamente el $60 \%$ de los individuos tenían el pie dominante de tipo normal, mientras que el pie no dominante era de tipo cavo (50,3\%); es decir, los mismos sujetos presentaron diferentes tipologías del pie. En cuanto a la sensibilidad plantar, hubo una diferencia en la región del mediopié durante el entrenamiento ( $p=0,001$ en el pie dominante y $p=0,009$ en el pie no dominante). Estos resultados demuestran que hubo un aumento en la presión media y el área total de contacto del pie, así como cambios en la sensibilidad plantar en la región del mediopié durante el servicio militar obligatorio.

Palabras clave | Pie; Presión; Mecanorreceptores.

\section{INTRODUÇÃO}

As lesões musculoesqueléticas são uma das principais causas de incapacidade e inaptidão dos soldados para serem efetivados na carreira militar ${ }^{1}$. Especialmente em jovens recrutas, um alto índice de lesões de membros inferiores tem sido relatado ${ }^{2-5}$. As atividades de risco para essas lesões incluem as corridas e marcha em longas distâncias, associadas às tarefas fisicamente exigentes como o transporte de cargas adicionais dos equipamentos de combate ${ }^{4,5}$.

A fadiga de marchas de longa-distância em combinação com transporte de cargas é considerada um importante fator de risco para lesões de sobrecarga dos membros inferiores em militares ${ }^{4,5}$. $\mathrm{O}$ transporte de cargas extras aumenta a pressão plantar no pé6,7, o que aumenta o estresse ósseo acumulado no local, predispondo à lesões ${ }^{8}$. Parâmetros como arco elevado ${ }^{9}$, aumento na carga nos metatarsos ${ }^{6,10,11}$ e maiores picos de pressão no calcanhar ${ }^{12}$ foram identificados como fatores de risco para fraturas.

As bolhas nos pés também são lesões comuns na marcha de soldados ${ }^{4,13}$ e qualquer alteração na rigidez na sola dos pés reduz a sensibilidade da região ${ }^{14,15}$. A redução na sensibilidade cutânea plantar altera parâmetros na distribuição da pressão plantar ${ }^{16,17}$, e os parâmetros de marcha também são alterados como mecanismo protecional ${ }^{18,19}$. Esta mudança na distribuição da carga pode agravar desequilíbrios préexistentes no pé, aumentando o risco de lesões em membros inferiores ${ }^{20}$.

Em recrutas, o risco das lesões de membro inferior pode ser ainda maior considerando que muitos deles passam por aumento rápido no nível de atividade física durante o treinamento militar, representando em muitos casos o primeiro contato dos indivíduos jovens com atividade física regular ${ }^{4}$. Tendo em vista que existe associação entre mudanças em variáveis de pressão e tipo de pé com um maior risco de lesões em recrutas militares ${ }^{9,12}$, quantificar as mudanças nessas variáveis e na sensibilidade plantar é importante para avaliar a influência de atividades físicas de alta demandas nessas características. O objetivo deste estudo foi avaliar os parâmetros da pressão plantar, tipo e sensibilidade do pé em recrutas durante o período do serviço militar obrigatório.

\section{METODOLOGIA}

Este é um estudo do tipo longitudinal observacional. A amostra foi obtida por conveniência e composta por 82 jovens recrutados para o serviço militar de um Esquadrão de Cavalaria Mecanizada, ingressantes no serviço militar obrigatório no Exército Brasileiro em março de 2018. O estudo foi aprovado pelo comitê de Ética e Pesquisa e seguiu as normas da Resolução CNS $n^{\circ} 466 / 2012$. Todos os sujeitos aceitaram participar do estudo e assinaram o Termo de Consentimento Livre e Esclarecido. Foram excluídos os participantes que já tinham realizado cirurgias e/ou apresentaram lesões moderadas e severas na região de quadril, joelho ou tornozelo, ou que apresentassem esta gravidade de lesão ao longo do estudo. Assim, na primeira avaliação foram investigadas as queixas musculoesqueléticas pelo questionário Nórdico de Sintomas Osteomusculares ${ }^{21}$, bem como as lesões e cirurgias prévias em membros inferiores por meio de uma entrevista estruturada desenvolvida para a pesquisa. As lesões prévias foram classificadas de acordo com o período de afastamento de atividades físicas: lesões menores correspondem ao afastamento por 1 a 7 dias, moderadas de 8 a 28 dias e 
severas são as lesões que afastaram da atividade por um período superior a 28 dias $^{22}$.

Nesta primeira etapa houve a exclusão de três sujeitos por já terem apresentado lesão ligamentar no joelho, sendo avaliados setenta e nove recrutas. Ao longo do estudo houve perda amostral adicional de 19 sujeitos, dois por baixa no serviço militar e 17 por não comparecerem a uma das avaliações subsequentes. Desta forma, a amostra foi composta por 60 indivíduos, com idade de 18,14 $\pm 0,35$ anos e índice de massa corporal (IMC) eutrófico, sem histórico de lesões, cirurgias prévias ou alterações significativas em membros inferiores, pés e tornozelo segundo o questionário Nórdico ${ }^{21}$.

Ao ingressarem para o exército, os recrutas realizam o treinamento físico militar de quatro a cinco vezes por semana. Esse treinamento é subdividido em fases introdutória, básico, qualificação e adestramento- de forma que cada fase segue uma progressão de carga e volume para melhora cardiorrespiratória, de resistência e força muscular conforme o manual de treinamento físico e militar do exército ${ }^{23}$. Nas três primeiras fases, há uma exigência física maior do recruta para que o mesmo esteja apto as demandas militares. Já na última etapa do treinamento (adestramento), os exercícios físicos são realizados em uma intensidade e volume menores, preparando o recruta para o desligamento do serviço militar. Baseados nessas fases, três avaliações foram realizadas após cada uma das três fases iniciais do treinamento. Assim, os sujeitos foram avaliados em três momentos ao longo do período de treinamento físico militar: março de 2018 (linha de base- AV1), junho de 2018 (após 16 semanas de treinamento- AV2) e setembro de 2018 (após 36 semanas de treinamentoAV3). Todas as avaliações foram realizadas pelos mesmos avaliadores previamente treinados para o procedimento de coleta.

Para a avaliação do IMC foi usado uma balança aferida com estadiômetro (marca Welmy, W2000A, Brasil), com resolução de $0,5 \mathrm{~cm}$ para medida da estatura e $0,1 \mathrm{~kg}$ para a medida da massa corporal. $\mathrm{Na} A V 1$, os recrutas foram questionados quanto a lesões, cirurgias prévias e sintomas osteomusculares por meio de uma entrevista estruturada e pelo Questionário Nórdico de Sintomas Osteomusculares ${ }^{21}$. Esse instrumento consiste em escolhas múltiplas ou binárias quanto à ocorrência de sintomas osteomusculares em todas as áreas anatômicas do corpo, considerando os 12 meses e os sete dias precedentes à entrevista, bem como afastamento de atividades de rotina.
Os recrutas que apresentassem lesões com afastamento de atividades por um período superior a 28 dias foram considerados com lesões graves em membros inferiores e excluídos do estudo ${ }^{22}$.

Nesta avaliação inicial também foi aplicado o Questionário Internacional de Atividade Física (IPAQ) para identificação dos níveis de atividades físicas prévio dos recrutas ${ }^{24}$. O IPAQ é um questionário com 27 questões que permite estimar o tempo semanal gasto em atividades físicas de intensidade moderada e vigorosa, em diferentes contextos como: trabalho, transporte, tarefas domésticas e lazer, e ainda o tempo despendido em atividades passivas realizadas na posição sentada. Posteriormente essas informações são utilizadas para cálculo em fórmulas específicas para cada seção de atividade física (trabalho, transporte, tarefas domésticas, lazer e esportes), com resultados expressos em equivalentes metabólicos (METs) em três níveis de atividade: caminhada, moderada e vigorosa. Esses resultados então produzem um escore final classificando o respondente nos níveis de atividade física baixo, moderado e alto ${ }^{24}$. Para o cálculo dos escores dos recrutas, a versão impressa foi computada na versão eletrônica do questionário (http://www.webipaq.com.br).

Para avaliação da sensibilidade ao toque superficial do pé utilizou-se o conjunto de monofilamentos de SemmesWeinstein com 6 monofilamentos. A sensibilidade plantar foi analisada de acordo com a classificação de espessura dos monofilamentos, permitindo um escore numérico para cada cor do fio: $0,05 \mathrm{~g}$ para o verde; $0,2 \mathrm{~g}$ para o azul; 2,0 g para o violeta; 4,0 g para o vermelho escuro; $10 \mathrm{~g}$ para o laranja e $300 \mathrm{~g}$ para o vermelho magenta. Quanto maior a espessura do fio, menor a capacidade de percepção sensitiva local e, consequentemente, menor a sensibilidade. A sensibilidade plantar foi avaliada com os recrutas deitados em supino e instruídos que, quando o filamento fosse colocado em pontos do pé, eles deveriam informar ao examinador onde eles sentiam. A ordem da avaliação das regiões do pé foi aleatória entre os sujeitos ${ }^{25}$ e pontos específicos na região plantar foram pressionados. Esses pontos são recomendados para avaliação do comprometimento da sensibilidade tátil superficial nas regiões do pé ${ }^{25,26}$. Na superfície plantar no ponto medial do retropé; no mediopé entre o arco medial e arco lateral; nas $1^{\mathrm{a}}, 3^{\mathrm{a}}$ e $5^{\mathrm{a}}$ cabeças metatársicas e no $1^{\circ}, 3^{\circ}$ e $5^{\circ}$ dedo do pé (Figura $\left.1 \mathrm{~A}\right)$. Para determinar a sensibilidade de antepé, médio pé e retropé (Figura 1B), a média dos valores encontrados nos pontos de cada região foi analisada. 


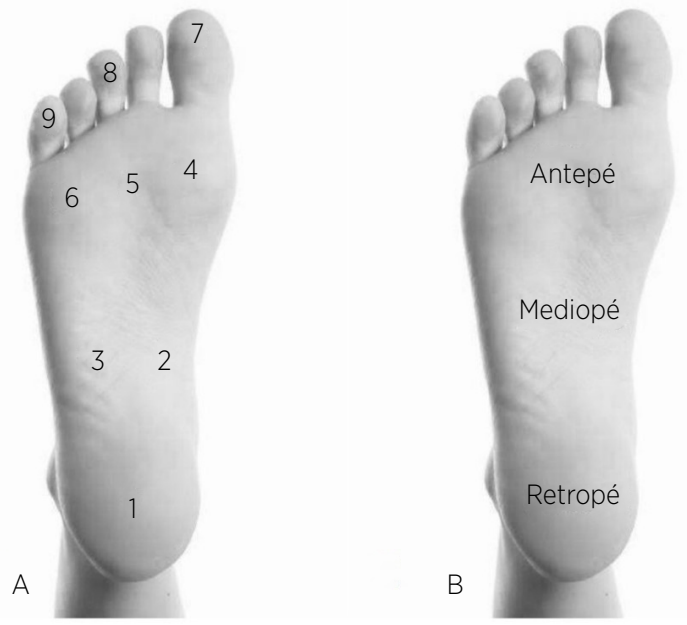

Figura 1. Ilustração dos Pontos de avaliação da sensibilidade (A) na superfície plantar entre o ponto medial do retropé (1); entre

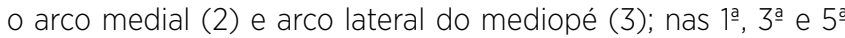

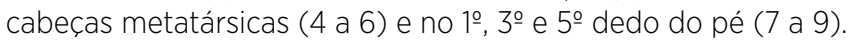
A ilustração B apresenta as subdivisões do pé utilizadas para a determinação da sensibilidade por região do pé

Para a mensuração dos parâmetros das pressões plantares foi utilizado um sistema de baropodometria eletrônica (EPS-R1 Loran, Loran Engineering, Bolonha, Itália). Este sistema contém superfície ativa de $480 \times 480$ $\mathrm{mm}$, dimensões de $675 \times 540 \times 5 \mathrm{~mm}, 2304$ sensores resistivos e frequência de aquisição de até $100 \mathrm{~Hz}$. Os sujeitos permaneceram descalços, em posição estática, com os pés alinhados na largura do quadril e os membros superiores ao longo do corpo. Os recrutas realizaram o teste de olhos abertos, com a cabeça direcionada a frente e olhando fixo em um ponto marcado a um metro de distância, na altura do ponto glabelar, por um período de 20 segundos. Cada sujeito realizou o teste três vezes, e o valor médio das três repetições foi utilizado para análise. Os dados foram analisados por meio do software Biomech ${ }^{\circledR}$ e as características avaliadas foram tipo de pé (normal, plano ou cavo) e distribuição plantar (antero-posterior e latero-lateral). As variáveis de distribuição plantar foram apresentadas em porcentagem
(\%), pressões média e máxima $(\mathrm{kPa})$ e área de contato do pé $\left(\mathrm{cm}^{2}\right)$.

\section{Análise estatística}

A análise estatística foi realizada com o programa Statistical Package for the Social Sciences Windows v.22.0 (SSPS, Chicago, IL, USA). A normalidade das variáveis foi avaliada pelo teste de Shapiro-Wilk. Para comparação das variáveis paramétricas foi usado a Anova de medidas repetidas e para variáveis não paramétricas foi usado o teste de Friedman. Para variáveis categóricas, a comparação entre os dados foi realizada utilizando o teste do Qui-quadrado. Havendo diferenças entre as avaliações, foi utilizado o teste de post hoc de Bonferoni. O nível de significância adotado foi de $5 \%(\mathrm{p}<0,05)$.

\section{RESULTADOS}

A Tabela 1 apresenta os dados antropométricos dos recrutas. Houve aumento da massa corporal dos recrutas da terceira em relação à primeira e segunda avaliação $(\mathrm{p}<0,001)$, o que se refletiu também em uma diferença no IMC $(p=0,008)$. Quanto à presença de alterações musculoesqueléticas na AV1, dos 60 recrutas avaliados $18,64 \%$ relataram dor em joelhos e $15,25 \%$ dor em tornozelo/pé. Além disso, 75\% apresentavam um nível de atividade física alto $(n=45), 15 \%$ moderado $(n=9)$ e $10 \%$ um nível baixo $(n=6)$.

Os resultados das variáveis baropodométricas estão apresentados na Tabela 2 . Na variável área de contato total do pé, houve redução da área na AV2 em relação a AV1 e aumento na área na AV2 para AV3 nos dois pés $(p=0,001)$. Além disso, houve aumento da pressão média de ambos os pés na AV1 em relação a AV3 $(\mathrm{p}<0,001)$.

Tabela 1. Características antropométricas dos 60 recrutas avaliados em três momentos ao longo do primeiro ano de treinamento físico militar: linha de base (AV1), após 16 semanas de treinamento (AV2) e após 36 semanas de treinamento (AV3). Dados apresentados em média \pm desvio padrão

\begin{tabular}{lrrrr} 
& \multicolumn{1}{c}{ AV1 } & \multicolumn{1}{c}{ AV2 } & AV3 & P \\
Massa corporal $(\mathrm{kg})$ & $71,78 \pm 14,1^{\mathrm{a}}$ & $72,17 \pm 11,72^{\mathrm{b}}$ & $73,23 \pm 12,42$ & 0,000 \\
Estatura $(\mathrm{m})$ & $1,71 \pm 0,06$ & $1,71 \pm 0,06$ & $1,71 \pm 0,05$ & 0,153 \\
IMC $\left(\mathrm{kg} / \mathrm{m}^{2}\right)$ & $24,30 \pm 4,22^{\mathrm{a}}$ & $24,61 \pm 3,29^{\mathrm{b}}$ & $24,92 \pm 3,61$ & 0,008
\end{tabular}

IMC: índice de massa corporal; adiferença significativa entre AV1 e AV3; bdiferença entre AV2 e AV3. 
Tabela 2. Variáveis baropodométricas dos pés dominante (D) e não dominante (ND) dos 60 recrutas avaliados em três momentos ao longo do primeiro ano de treinamento físico militar: linha de base (AV1), após 16 semanas de treinamento (AV2) e após 36 semanas de treinamento (AV3). Dados apresentados em mediana (25\%-75\%)

\begin{tabular}{|c|c|c|c|c|}
\hline & AV1 & AV2 & AV3 & $\mathbf{P}$ \\
\hline Descarga anterior (\%) & $\begin{array}{r}49,2 \\
(46,24-52,95)\end{array}$ & $\begin{array}{r}50,12 \\
(44,33-58,03)\end{array}$ & $\begin{array}{r}47,92 \\
(42,10-56,05)\end{array}$ & 0,265 \\
\hline Descarga posterior (\%) & $\begin{array}{r}50,8 \\
(43,14-53,75)\end{array}$ & $\begin{array}{r}49,88 \\
(41,97-55,39)\end{array}$ & $\begin{array}{r}52,06 \\
(43,94-57,89)\end{array}$ & 0,281 \\
\hline Descarga lateral D (\%) & $\begin{array}{r}49,92 \\
(48,53-51,54)\end{array}$ & $\begin{array}{r}49,73 \\
(47,73-52,10)\end{array}$ & $\begin{array}{r}49,76 \\
(47,49-51,95)\end{array}$ & 0,803 \\
\hline Descarga lateral ND (\%) & $\begin{array}{r}50,08 \\
(48,45-51,47)\end{array}$ & $\begin{array}{r}50,27 \\
(47,89-55,52)\end{array}$ & $\begin{array}{r}50,23 \\
(48,05-52,51)\end{array}$ & 0,647 \\
\hline Área total do pé $\mathrm{D}\left(\mathrm{cm}^{2}\right)$ & $\begin{array}{r}106,33 \\
(95-119)\end{array}$ & $\begin{array}{r}102,67 \\
(91-117,91)^{\mathrm{bc}}\end{array}$ & $\begin{array}{r}104,16 \\
(93-116,91)\end{array}$ & 0,001 \\
\hline Área total do pé ND $\left(\mathrm{cm}^{2}\right)$ & $\begin{array}{r}104,83 \\
(94,9-115,6)\end{array}$ & $\begin{array}{r}99,83 \\
(90,83-113,5)^{b c}\end{array}$ & $\begin{array}{r}105,83 \\
(92,41-116)\end{array}$ & 0,001 \\
\hline Pressão média D (kPa) & $\begin{array}{r}61,43 \\
(57,42-71,10)^{a}\end{array}$ & $\begin{array}{r}64,05 \\
(58,31-74,42)\end{array}$ & $\begin{array}{r}64,80 \\
(60,23-74,82)\end{array}$ & $<0,001$ \\
\hline Pressão média ND (kPa) & $\begin{array}{r}62,47 \\
(57,99-70,47)^{a}\end{array}$ & $\begin{array}{r}68,78 \\
(57,51-75,25)\end{array}$ & $\begin{array}{r}69,40 \\
(59,01-76,04)\end{array}$ & $<0,001$ \\
\hline Pressão máxima D (kPa) & $\begin{array}{r}238,32 \\
(182,9-284,6)\end{array}$ & $\begin{array}{r}231,38 \\
(205-263,2)\end{array}$ & $\begin{array}{r}235,17 \\
(185,72-267,4)\end{array}$ & 0,475 \\
\hline Pressão máxima ND (kPa) & $\begin{array}{r}232,95 \\
(183,39-276,5)\end{array}$ & $\begin{array}{r}225,01 \\
(193-254,8)\end{array}$ & $\begin{array}{r}222,98 \\
(198,2-275,5)\end{array}$ & 0,628 \\
\hline
\end{tabular}

adiferença significativa entre AV1 e AV3; bdiferença entre AV2 e AV3; cdiferença entre AV1 e AV2.

A Tabela 3 apresenta o tipo de pé dos recrutas ao longo das avaliações. A maioria dos recrutas apresentou o pé dominante do tipo normal, enquanto o pé não dominante teve uma frequência semelhante entre os tipos normal e cavo. A comparação das frequências dos tipos de pé não foi diferente entre as três avaliações realizadas tanto para o pé dominante $(\mathrm{p}=0,051)$ como para o pé não dominante $(\mathrm{p}=0,998)$, demonstrando estabilidade dessa variável.
Quanto a avaliação da sensibilidade plantar, houve mudanças na região do médio pé dominante $(\mathrm{p}=0,001)$ e não-dominante $(\mathrm{p}=0,009)$. No pé dominante ocorreu uma melhora da sensibilidade na AV2 em relação a AV1 e piora na AV2 em relação a $A V 3$, retornando aos valores da avaliação basal (AV1). Já para o pé não-dominante, houve uma piora na sensibilidade da AV1 para AV3. Mesmo com essa diferença entre os pés, a sensibilidade permaneceu preservada com valores entre 0,05 e 0,2 $\mathrm{g}$ (Tabela 4).

Tabela 3. Tipo de pé dos 60 recrutas avaliados em três momentos ao longo do primeiro ano de treinamento físico militar: linha de base (AV1), após 16 semanas de treinamento (AV2) e após 36 semanas de treinamento (AV3). Dados apresentados em valor absoluto (percentual)

\begin{tabular}{|c|c|c|c|c|c|}
\hline & & AV1 & AV2 & AV3 & $\mathbf{P}$ \\
\hline \multirow{3}{*}{ Dominante } & Normal & $40(66,7)$ & $35(58,3)$ & $36(60)$ & \multirow{3}{*}{0,051} \\
\hline & Cavo & $30(18)$ & $19(31,7)$ & $17(28,3)$ & \\
\hline & Plano & $2(3,3)$ & $6(10)$ & $7(11,7)$ & \\
\hline \multirow{3}{*}{ Não dominante } & Normal & $27(45)$ & $26(43,3)$ & $27(45)$ & \multirow{3}{*}{0,998} \\
\hline & Cavo & $30(50)$ & $31(51,7)$ & $30(50)$ & \\
\hline & Plano & $3(5)$ & $3(5)$ & $3(5)$ & \\
\hline
\end{tabular}

Tabela 4. Valores da sensibilidade plantar dos pés dominante (D) e não dominante (ND) de 60 recrutas militares avaliados em três momentos ao longo do primeiro ano de treinamento físico militar: linha de base (AV1), após 16 semanas de treinamento (AV2) e após 36 semanas de treinamento (AV3). Dados apresentados em mediana (25\%- 75\%) de gramas (g)

\begin{tabular}{lrrrr} 
& \multicolumn{1}{c}{ AV1 } & AV2 & \multicolumn{1}{c}{ AV3 } & p \\
Antepé D & $0,15(0,1-0,2)$ & $0,12(0,05-0,2)$ & $0,17(0,1-0,2)$ & 0,057 \\
Antepé ND & $0,13(0,1-0,2)$ & $0,14(0,05-0,2)$ & $0,17(0,1-0,2)$ & 0,121 \\
Médiopé D & $0,10(0,05-0,18)$ & $0,05(0-0,12)^{b c}$ & $0,10(0,05-0,2)$ & 0,001 \\
Médiopé ND & $0,10(0,05-0,2)$ & $0,10(0-0,12)^{c}$ & $0,12(0,05-0,2)$ & 0,009 \\
Retropé D & $0,20(0,08-0,2)$ & $0,20(0,05-0,2)$ & $0,20(0,2-0,2)$ & 0,140 \\
Retropé ND & $0,20(0,2-0,2)$ & $0,20(0,2-0,2)$ & $0,20(0,2-0,2)$ & 0,144
\end{tabular}

bdiferença entre AV2 e AV3; cdiferença entre AV1 e AV2. 


\section{DISCUSSÃO}

O objetivo principal desse trabalho foi investigar se os parâmetros de pressão plantar, tipo e sensibilidade do pé apresentariam alterações em jovens recrutas após o início do treinamento militar. É importante destacar que os sujeitos eram fisicamente ativos ao ingressarem no serviço militar e sem lesões significativas de membros inferiores segundo os questionários IPAQ e Nórdico. Este fato pode ter auxiliado os recrutas na transição para o treinamento físico militar, uma vez que já possuíam um estilo de vida que envolvia a prática de atividades físicas e esportivas. Além disso, os valores de IMC na primeira avaliação estavam dentro dos limites de normalidade. Embora não se tenha avaliado a composição corporal, é presumível que esse aumento significativo ao longo do período de treinamento tenha ocorrido pelo aumento da massa muscular, consequência do tipo de treinamento a que foram expostos ${ }^{27}$.

A avaliação da distribuição da pressão plantar ânteroposterior evidenciou que os sujeitos apresentaram valores de descarga de peso anterior acima e posterior abaixo da normalidade, conforme os valores do estudo de Pormarino e Pormarino ${ }^{28}$ que correspondem a aproximadamente $40 \%$ anterior e $60 \%$ posterior. Esses valores estão mais discrepantes na AV2, o que sugere um processo de adaptação física que pode estar relacionado a corridas de longa distância e também ao uso de cargas adicionais de equipamentos militares especialmente nessas primeiras 16 semanas de treinamento físico militar. Segundo Andersen et al. ${ }^{4}$, em geral um peso entre $20-68 \mathrm{~kg}$ pode ser considerado como uma carga típica de equipamento adicional que os soldados precisam carregar regularmente. Estudos prévios sugerem alterações nesses parâmetros na postura estática em pé em recrutas após atividade militar com transporte de carga prolongada ${ }^{29}$ e em maratonistas após corridas de longa distância ${ }^{10}$.

Quanto à área de contato do pé, houve mudanças ao longo das avaliações com redução na segunda avaliação para a primeira e aumento para a terceira. Apesar dessas alterações, os valores permaneceram em aproximadamente $100 \mathrm{~cm}^{2}$, o que pode ser considerado dentro da normalidade ${ }^{30}$. Essas mudanças na área de contato podem induzir a redistribuição da pressão plantar sob o pé, aumentando o pico de pressão em outros locais ${ }^{31}$, o que tem sido associado a fraturas no pé e tornozelo especialmente em recrutas militares ${ }^{9,12}$. Em nosso estudo houve um aumento gradativo nos valores de pressão média no decorrer das avaliações, o que pode aumentar a exposição dos recrutas aos riscos de lesões durante o treinamento militar obrigatório.

Quanto à tipologia de pé, observou-se um predomínio no pé dominante do tipo normal, enquanto o não dominante foi do tipo normal e também cavo. Os pés cavos apresentam uma tendência a menor movimento de suas estruturas, maiores pressões no retropé e região lateral do antepé, diminuindo a sua capacidade de atenuação do choque $^{32}$. As características da pressão plantar também são diferentes conforme cada tipo de pé $\mathrm{e}^{32}$, o que pode explicar os diferentes valores nas pressões plantares encontrados neste estudo, considerando as diferenças tipológicas do pé no mesmo sujeito.

As diferenças das avaliações na sensibilidade plantar no médio pé demonstraram preservação da sensibilidade do lado dominante e redução no lado não dominante ao final das 36 semanas de treinamento. Apesar dessas mudanças, os recrutas apresentaram uma sensibilidade dentro da normalidade ( 0,05 a 0,2 g), correspondendo aos dois primeiros monofilamentos de avaliação $0^{25,26}$. Assim, as diferenças na distribuição da pressão plantar não podem ser atribuídas às alterações de sensibilidade plantar.

Uma limitação de nosso estudo é não ter realizado um grupo controle sem atividades físicas extenuantes para avaliação dessas variáveis. Além disso, não houve o questionamento sobre o uso habitual de outros calçados ou de atividades físicas paralelas ao treinamento físico militar. Entretanto, considerando que os recrutas permanecem a maior parte do dia no quartel realizando atividades durante o período de serviço obrigatório, pode-se considerar que os recrutas dessa amostra utilizaram o mesmo calçado e realizaram as mesmas atividades durante pelo menos 36 horas semanais.

$\mathrm{O}$ treinamento físico militar produziu mudanças nos parâmetros baropodométricos e de sensibilidade plantar. Houve diferença na área de contato do pé e pressão média, bem como valores superiores de descarga anterior. Na sensibilidade plantar, a região do médio pé apresentou preservação da sensibilidade do lado dominante e redução no lado não dominante. Essas mudanças ocorreram essencialmente após as primeiras semanas do treinamento, uma fase de maior exigência física e de adaptação a rotina militar. Acredita-se que essas mudanças ocorrem pela intensidade dos exercícios físicos no treinamento militar e podem aumentar o risco de lesões nessa população. 


\section{CONCLUSÃO}

O treinamento físico militar obrigatório gera alterações nas características baropodométricas e na sensibilidade plantar em recrutas. Há aumento da pressão média e área total de contato do pé, bem como alterações na sensibilidade plantar da região do médio pé ao longo de 36 semanas de serviço militar obrigatório.

\section{REFERÊNCIAS}

1. Hauret KG, Bedno S, Loringer K, Kao TC, Mallon T, Jones BH. Epidemiology of exercise- and sports-related injuries in a population of young, physically active adults: a survey of military servicemembers. Am J Sports Med. 2015;43(11):2645-53. doi: 10.1177/0363546515601990

2. Lopes TJA, Simic M, Bunn PS, Terra BS, Alves DS, Ribeiro FM, et al. Prevalence of musculoskeletal symptoms among Brazilian merchant navy cadets: differences between sexes and school years. Mil Med. 2017;182(11):1987-72. doi: 10.7205/ milmed-d-17-00124

3. Gomes MZA, Pinfildi CE. Prevalence of musculoskeletal injuries and a proposal for neuromuscular training to prevent lower limb injuries in Brazilian Army soldiers: an observational study. Mil Med Res. 2018;5(1):1-7. doi: 10.1186/s40779-018-0172-7

4. Andersen KA, Grimshaw PN, Kelso RM, Bentley DJ. Musculoskeletal lower limb injury risk in army populations. Sport Med Open. 2016;2:22. doi: 10.1186/s40798-016-0046-z

5. Orr RM, Johnston V, Coyle J, Pope R. Reported load carriage injuries of the Australian Army soldier. J Occup Rehabil. 2015;25(2):316-22. doi:10.1007/s10926-014-9540-7

6. Arndt A, Ekenman I, Westblad P, Lundberg A. Effects of fatigue and load variation on metatarsal deformation measured in vivo during barefoot walking. J Biomech. 2002;35(5):621-8. doi: 10.1016/S0021-9290(01)00241-X

7. Goffar SL, Reber RJ, Christiansen BC, Miller RB, Naylor JA, Rodriguez BM, et al. Changes in dynamic plantar pressure during loaded gait. Phys Ther. 2013;93(9):1175-84. doi: 10.2522/ptj.20120103

8. Carter DR, Caler WE, Spengler DM, Frankel VH. Fatigue behavior of adult cortical bone: the influence of mean strain and strain range. Acta Orthop Scand. 1981;52(5):481-90. doi: 10.3109/17453678108992136

9. Dixon S, Nunns M, House C, Rice H, Mostazir M, Stiles V, et al. Prospective study of biomechanical risk factors for second and third metatarsal stress fractures in military recruits. J Sci Med Sport. 2019;22(2):135-9. doi: 10.1016/j.jsams.2018.06.015

10. Nagel A, Fernholz F, Kibele C, Rosenbaum D. Long distance running increases plantar pressures beneath the metatarsal heads. Gait Posture. 2008;27(1):152-5. doi: 10.1016/ j.gaitpost.2006.12.012

11. Rosenbaum D, Engl T, Nagel A. Effects of a fatiguing longdistance run on plantar loading during barefoot walking and shod running. Footwear Sci. 2016;8(3):129-37. doi: 10.1080/19424280.2016.1157103

12. Nunns M, House C, Rice H, Mostazir M, Davey T, Stiles V, et al. Four biomechanical and anthropometric measures predict tibial stress fracture: a prospective study of 1065 Royal Marines. Br J Sports Med. 2016;50(19):1206-10. doi: 10.1136/ bjsports-2015-095394

13. Reynolds KL, White JS, Knapik JJ, Witt CE, Amoroso PJ. Injuries and risk factors in a 100-mile (161-km) infantry road march. Prev Med. 1999;28(2):167-73. doi: 10.1006/pmed.1998.0396

14. Jammes Y, Ferrand E, Fraud C, Boussuges A, Weber JP. Adding body load modifies the vibratory sensation of the foot sole and affects the postural control. Mil Med Res. 2018;5(1):28. doi: 10.1186/s40779-018-0175-4

15. Jammes Y, Viala M, Dutto W, Weber JP, Guieu R. Skin Hardness and Epidermal Thickness Affect the Vibration Sensitivity of the Foot Sole. Clin Res Foot Ankle. 2017;5(3):1-5. doi: 10.4172/2329-910x.1000245

16. Chen $H$, Nigg BM, Hulliger M, de Koning J. Influence of sensory input on plantar pressure distribution. Clin Biomech. 1995;10(5):271-4. doi: 10.1016/0268-0033(95)99806-D

17. Nurse MA, Nigg BM. Quantifying a relationship between tactile and vibration sensitivity of the human foot with plantar pressure distributions during gait. Clin Biomech. 1999;14(9):667-72. doi: 10.1016/S0268-0033(99)00020-0

18. Eils E, Nolte S, Tewes M, Thorwesten L, Völker K, Rosenbaum D. Modified pressure distribution patterns in walking following reduction of plantar sensation. J Biomech. 2002;35(10):1307-13. doi: 10.1016/S0021-9290(02)00168-9

19. Hohne A, Ali S, Stark C, Bruggemann GP. Reduced plantar cutaneous sensation modifies gait dynamics, lower-limb kinematics and muscle activity during walking. Eur J Appl Physiol. 2012;112(11):3829-38. doi: 10.1007/s00421-012-2364-2

20. Bush RA, Brodine SK, Shaffer RA. The association of blisters with musculoskeletal injuries in male marine recruits. J Am Podiatr Med Assoc. 2000;90(4):194-8. doi: 10.7547/87507315-90-4-194

21. De Barros ENC, Alexandre NMC. Cross-cultural adaptation of the Nordic musculoskeletal questionnaire. Int Nurs Rev. 2003:50(2):101-8. doi: 10.1046/j.1466-7657.2003.00188.x

22. Junge A, Engebretsen L, Alonso JM, Renström P, Mountjoy M, Aubry $M$, et al. Injury surveillance in multi-sport events: The International Olympic Committee approach. Br J Sports Med. 2008;42(6):413-21. doi:10.1136/bjsm.2008.046631

23. Brasil. Ministério da Defesa. Exército Brasileiro. Manual de campanha: treinamento físico militar. 4th ed. Brasília, DF; 2015.

24. Matsudo S, Araújo T, Matsudo V, Andrade D, Andrade E, Oliveira LC, et al. Questionário Internacional de Atividade Física (Ipaq): estudo de validade e reprodutibilidade no Brasil. Rev Bras Ativ Fis e Saude. 2001;6(2):5-18. doi: 10.12820/rbafs.v.6n2p5-18

25. Hennig EM, Sterzing T. Sensitivity mapping of the human foot: thresholds at 30 skin locations. Foot Ankle Int. 2009;30(10):98691. doi: 10.3113/FAl.2009.0986

26. Machado ÁS, Bombach GD, Duysens J, Carpes FP. Differences in foot sensitivity and plantar pressure between young adults and elderly. Arch Gerontol Geriatr. 2016;63:67-71. doi: 10.1016/j.archger.2015.11.005 
27. Avila JA, Lima Filho PDB, Páscoa MA, Tessutti LS. Efeito de 13 semanas de treinamento físico militar sobre a composição corporal e o desempenho físico dos alunos da escola preparatória de cadetes do exército. Rev Bras Med do Esporte. 2013;19(5):363-6. doi: 10.1590/S1517-86922013000500013

28. Pomarino D, Pomarino A. Plantar static pressure distribution in healthy individuals: percentiles for the evaluation of forefoot loading. Foot Ankle Spec. 2014;7(4):293-7. doi: 10.1177/1938640014528973

29. Rice H, Fallowfield J, Allsopp A, Dixon S. Altered forefoot function following a military training activity. Gait Posture. 2019;74:182-6. doi: 10.1016/j.gaitpost.2019.09.009
30. Nazario PF, Santos JOL, Avila AOV. Comparação da distribuição de pressão plantar em sujeitos com pés normais e com pés planos durante a marcha. Rev Bras Cineantropom Desempenho Hum. 2011;12(4):290-4. doi: 10.5007/1980-0037.2010v12n4p290

31. Sneyers CJL, Lysens R, Feys H, Andries R. Influence of malalignment of feet on the plantar pressure pattern in running. Foot Ankle Int. 1995;16(10):624-32. doi: 10.1177/107110079501601008

32. Buldt AK, Allan JJ, Landorf KB, Menz HB. The relationship between foot posture and plantar pressure during walking in adults: a systematic review. Gait Posture. 2018;62:56-67. doi: 10.1016/j.gaitpost.2018.02.026 